\title{
Erratum
}

\section{Three Conjectures of Combinatorial Topology}

\author{
M. Gutierrez
}

Invent. math. 67, 357-362 (1982)

There are serious errors in the proofs of Lemmas 1 and 6 , on which the entire paper depends. The results of this article, therefore, are not proved and should be considered open problems. 